\title{
Multi-orbital cluster dynamical mean-field theory with an improved continuous-time quantum Monte Carlo algorithm
}

\author{
Yusuke Nomura . Shiro Sakai, and Ryotaro Arita \\ Department of Applied Physics, University of Tokyo, Hongo, Bunkyo-ku, Tokyo, 113-8656, Japan.
}

(Dated: October 7, 2018)

\begin{abstract}
We implement a multi-orbital cluster dynamical mean-field theory (DMFT), by improving a sample-update algorithm in the continuous-time quantum Monte Carlo method based on the interaction expansion. The proposed sampling scheme for the spin-flip and pair-hopping interactions in the two-orbital systems mitigates the sign problem, giving an efficient way to deal with these interactions. In particular, in the single-site DMFT, we see that the negative signs vanish. We apply the method to the two-dimensional two-orbital Hubbard model at half filling, where we take into account the short-range spatial correlation effects within a four-site cluster. We show that, compared to the single-site DMFT results, the critical interaction value for the metal-insulator transition decreases and that the effects of the spin-flip and pair-hopping terms are less significant in the parameter region we have studied. The present method provides a firm starting point for the study of inter-site correlations in multi-orbital systems. It also has a wide applicable scope in terms of realistic calculations in conjunction with density functional theory.
\end{abstract}

PACS numbers: $71.27 .+\mathrm{a}, 02.70 . \mathrm{Tt}, 71.10 . \mathrm{Fd}$

\section{INTRODUCTION}

Strongly correlated materials have attracted much interest because of their diverse fascinating properties, $\frac{1}{1}$ which are believed to originate from a severe competition between the itinerancy and the locality of low-energy electrons. A minimal model to describe this competition is the Hubbard model, which has been found to be surprisingly versatile despite its simple definition. In two or three dimensions, the Hubbard model has not been solved analytically, except for several special cases $\stackrel{2}{\stackrel{2}{2} \text { and }}$ therefore we have to resort to numerical simulations.

The dynamical mean-field theory (DMFT),$\frac{3}{\underline{3}}$ which takes into account the dynamical local correlations accurately by mapping a lattice model onto a single impurity problem subject to a self-consistency condition, is one of the most successful methods for describing the strongcorrelation physics such as the Mott transition in infinite dimensions $\stackrel{3}{3}$ However, the DMFT totally neglects the spatial correlations, which are essential in quantitative and also qualitative description of real materials. For example, the single-site DMFT cannot describe the $d$ wave superconductivity observed in high- $T_{c}$ cuprates. To overcome this problem, cluster extensions of the DMFT

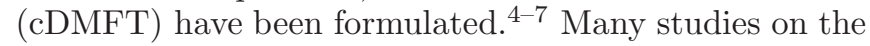
two-dimensional (2D) single-orbital Hubbard model have been performed by the cDMFT to clarify the pseudogap phase $e^{\frac{8}{2}-26}$ and the superconductivity $\underline{27-42}$ of the cuprates.

More generally, in most strongly correlated materials, several orbitals are involved in the low-energy region around the Fermi level, as exemplified by the transition metal compounds and heavy fermion systems. A description of these materials requires an extention of the Hubbard model to the multi-orbital one. Even in the cuprates, where orbitals other than the one composing the Fermi surface are neglected in many cases, it has been proposed that the orbital degrees of freedom play a key role $\underline{43}-\underline{45}$ in accounting for the material dependence of the superconducting transition temperature.

These manifest the importance of studying multiorbital Hubbard model with including the spatial correlations. Nevertheless, it has barely been explored before because of the huge computational cost in solving the impurity problem. A few exceptions are the 2-site cDMFT + the non-crossing approximation study of a two-orbital model in Ref. 46, the 2-site cDMFT + the Hirsch-Fye quantum Monte Carlo calculation 47 of a three-orbital model for $\mathrm{Ti}_{2} \mathrm{O}_{3}$ in Ref. 48, and the 4-site cDMFT + the continuous-time quantum Monte Carlo (CTQMC) $\underline{49,50}$ calculation for an anisotropic two-orbital model in Ref. 51. In the latter two studies, the spin-flip and pair-hopping terms present in the multi-orbital Hubbard Hamiltonian were neglected. A study based on an accurate numerical calculation on the full multi-orbital Hamiltonian (i.e., with the spin-flip and pair-hopping terms) is still missing in literature. Then, the aim of the present paper is to develop such a numerical scheme and to provide the first calculated results to explore the inter-site correlation physics in the multi-orbital systems.

In the present study, we adopt the CTQMC algorithm based on the interaction expansion (CT-INT) $\stackrel{52,53}{:}$ Compared to other CTQMC algorithms $\stackrel{49}{\underline{49}}$ the CT-INT has an advantage in incorporating various types of interactions such as Hund's coupling and electron-phonon interacton $\stackrel{54,55}{ }$ It also gives an efficient way to deal with relatively large degrees of freedom, complementary to the algorithm based on the hybridization expansion, $56-58$ which is efficient for a few degrees of freedom while the computational cost grows exponentially with the degrees of freedom. Moreover, an efficient sampling update algorithm, called submatrix update algorithm, has recently been developed for another weak-coupling CTQMC method exploiting an auxiliary-field decomposition (CT-AUX), and has been successfully employed 
in cDMFT calculations on the $2 \mathrm{D}^{21,22,24,40}$ and three dimensiona 160 single-orbital Hubbard models. As we will show in this work, a similar submatrix update algorithm can apply to the CT-INT as well as to the multi-orbital models, too, and it enables us to reach a strongly-correlated regime at rather low temperatures within the multi-orbital cDMFT in a reasonable computational time. Furthermore, we develop a sampling scheme which mitigates the sign problem coming from the spin-flip and pair-hopping terms in the two-orbital models. Although in the cDMFT the negative signs remain due to the one-body hopping terms within the cluster, in the single-site DMFT, we see that the proposed method completely eliminates the negative signs.

We apply the method to the 2D two-orbital Hubbard model on a square lattice within the 4-site cellular DMFT 5 We show that the short-range spatial correlations reduce the critical interaction strength of the Mott metal-insulator transition substantially. We also find that the model with the Ising-type Hund's coupling overestimates the tendency toward the insulating phase while the difference between the results with and without the spin-flip and pair-hopping terms is less significant than that of the single-site DMFT.

This paper is organized as follows. In Sec. II] we briefly review the CT-INT algorithm and show how the submatrix update and the efficient update scheme for the nondensity-density interactions are incorporated into the algorithm. We show the cellular DMFT results for the 2D two-orbital Hubbard model in Sec. III Section IV is devoted to the conclusion. The derivation of the several equations used in Sec. II] and a proof of the absence of negative signs in the two-orbital models in our scheme are given in Appendices.

\section{METHOD}

In this section, we explain, in detail, the schemes employed in our calculations. Sec. IA 1 and Sec. II 2 are devoted to a brief introduction of the CT-INT algorithm. Sec. IIA3 shows how the submatrix update scheme, which has been employed only in the Hirsch-Fye and CT-AUX algorithms in literature, is incorporated in the CT-INT method. In Sec. IIB, we show the extension to the single-site multi-orbital case, where we propose an efficient sampling scheme for the spin-flip and pairhopping terms, double-vertex update, in the two-orbital case. Finally, we show the extension to multi-site multiorbital case in Sec. IIC

\section{A. Single-orbital case}

\section{Interaction expansion of partition function}

The CT-INT algorithm was developed by Rubtsov et $a l .52,53$ Here we review the basic part of the algorithm in order to define our notations used in the next section. We first consider the single-orbital and single-impurity model for simplicity.

The action for the single-orbital impurity problem reads

$$
S_{\mathrm{imp}}=S_{0}+S_{\mathrm{int}}
$$

where

$$
S_{0}=-\int_{0}^{\beta} d \tau \int_{0}^{\beta} d \tau^{\prime} \sum_{\sigma} \mathcal{G}_{0 \sigma}^{-1}\left(\tau-\tau^{\prime}\right) \hat{c}_{\sigma}^{\dagger}(\tau) \hat{c}_{\sigma}\left(\tau^{\prime}\right)
$$

and

$$
S_{\mathrm{int}}=\int_{0}^{\beta} d \tau U \hat{n}_{\uparrow}(\tau) \hat{n}_{\downarrow}(\tau)
$$

with the inverse temperature $\beta$, the bath Green's function $\mathcal{G}_{0 \sigma}$, and the Hubbard interaction $U$. $\hat{c}_{\sigma}^{\dagger}\left(\hat{c}_{\sigma}\right)$ is a Grassmann variable representing the creation (annihilation) of an impurity electron with the spin $\sigma$, and $\hat{n}_{\sigma}=\hat{c}_{\sigma}^{\dagger} \hat{c}_{\sigma}$.

In order to reduce the sign problem, we introduce additional parameters $\alpha_{\sigma}$ defined as ${ }^{54}$

$$
\begin{aligned}
& \alpha_{\uparrow}(s)=1 / 2+s \delta, \\
& \alpha_{\downarrow}(s)=1 / 2-s \delta
\end{aligned}
$$

with $\delta=1 / 2+0^{+}$and $s= \pm 1$. In practice, we typically set $0^{+}$to be the order of $10^{-2}$. In the absence of this $\alpha$ term, we suffer from the negative sign problem because the elements of the $V$ matrix corresponding to the $U$ vertex in Eq. (B2) can take negative values, 61 Then the action is recast into

$$
S_{0}=-\int_{0}^{\beta} d \tau \int_{0}^{\beta} d \tau^{\prime} \sum_{\sigma} \tilde{\mathcal{G}}_{0 \sigma}^{-1}\left(\tau-\tau^{\prime}\right) \hat{c}_{\sigma}^{\dagger}(\tau) \hat{c}_{\sigma}\left(\tau^{\prime}\right)
$$

and

$$
S_{\text {int }}=\int_{0}^{\beta} d \tau \sum_{s= \pm 1} \frac{U}{2}\left[\hat{n}_{\uparrow}(\tau)-\alpha_{\uparrow}(s)\right]\left[\hat{n}_{\downarrow}(\tau)-\alpha_{\downarrow}(s)\right],(6)
$$

where $\tilde{\mathcal{G}}_{0 \sigma}$ is the Weiss function defined with a new chemical potential $\tilde{\mu}=\mu-U / 2$. The perturbation expansion with respect to $U$ term leads to 


$$
\begin{aligned}
\frac{Z}{Z_{0}} & =\sum_{n=0}^{\infty}\left(-\frac{U}{2}\right)^{n} \int_{0}^{\beta} d \tau_{1} \sum_{s_{1}= \pm 1} \cdots \int_{0}^{\tau_{n-1}} d \tau_{n} \sum_{s_{n}= \pm 1} \prod_{\sigma}\left\langle\left[\hat{n}_{\sigma}\left(\tau_{1}\right)-\alpha_{\sigma}\left(s_{1}\right)\right] \cdots\left[\hat{n}_{\sigma}\left(\tau_{n}\right)-\alpha_{\sigma}\left(s_{n}\right)\right]\right\rangle_{0} \\
& =\sum_{n=0}^{\infty}\left(-\frac{U}{2}\right)^{n} \int_{0}^{\beta} d \tau_{1} \sum_{s_{1}= \pm 1} \ldots \int_{0}^{\tau_{n-1}} d \tau_{n} \sum_{s_{n}= \pm 1} \prod_{\sigma} \operatorname{det} A_{\sigma}^{\prime}\left(\left\{s_{i}, \tau_{i}\right\}\right)
\end{aligned}
$$

where $Z_{0}=\int \mathcal{D}\left[\hat{c}^{\dagger}, \hat{c}\right] e^{-S_{0}\left[\hat{c}^{\dagger}, \hat{c}\right]}$ is a noninteracting partition function and the thermal average for the products of Grassmann variables $\left\langle V\left[\hat{c}^{\dagger}, \hat{c}\right]\right\rangle_{0}$ is defined as

$$
\left\langle V\left[\hat{c}^{\dagger}, \hat{c}\right]\right\rangle_{0}=\int \mathcal{D}\left[\hat{c}^{\dagger}, \hat{c}\right] e^{-S_{0}\left[\hat{c}^{\dagger}, \hat{c}\right]} V\left[\hat{c}^{\dagger}, \hat{c}\right] .
$$

$A_{\sigma}^{\prime}\left(\left\{s_{i}, \tau_{i}\right\}\right)$ is an $n \times n$ matrix whose element is given by

$$
\left[A_{\sigma}^{\prime}\left(\left\{s_{i}, \tau_{i}\right\}\right)\right]_{i j}=\tilde{\mathcal{G}}_{0 \sigma}\left(\tau_{i}-\tau_{j}\right)-\alpha_{\sigma}\left(s_{i}\right) \delta_{i j} .
$$

With a function

$$
f_{\sigma}(s)= \begin{cases}\frac{\alpha_{\sigma}(s)}{\alpha_{\sigma}(s)-1} & s= \pm 1 \\ 1 & s=0\end{cases}
$$

a configuration

$$
C_{n}=\left\{\left(s_{1}, \tau_{1}\right), \cdots,\left(s_{n}, \tau_{n}\right)\right\},
$$

Eq. (77) is rewritten as

$$
\begin{aligned}
\frac{Z}{Z_{0}}= & \sum_{n=0}^{\infty} \int_{0}^{\beta} \sum_{s_{1}= \pm 1} \cdots \int_{0}^{\tau_{n-1}} \sum_{s_{n}= \pm 1} \\
& {\left[\prod_{i=1}^{n} \frac{K\left(s_{i}\right) d \tau_{i}}{2 \beta} \times \prod_{\sigma} \operatorname{det} A_{\sigma}\left(C_{n}\right)\right], }
\end{aligned}
$$

where

$$
\begin{aligned}
& K(s)=\frac{-\beta U}{\left(f_{\uparrow}(s)-1\right)\left(f_{\downarrow}(s)-1\right)} \text { for } s= \pm 1, \\
& A_{\sigma}\left(C_{n}\right)=F_{\sigma}^{\left\{s_{i}\right\}}-G_{0 \sigma}^{\left\{\tau_{i}\right\}}\left(F_{\sigma}^{\left\{s_{i}\right\}}-1\right) .
\end{aligned}
$$

Here, we define $n \times n$ matrices $G_{0 \sigma}^{\left\{\tau_{i}\right\}}$ and $F_{\sigma}^{\left\{s_{i}\right\}}$, whose elements are

$$
\left[G_{0 \sigma}^{\left\{\tau_{i}\right\}}\right]_{i j}=\tilde{\mathcal{G}}_{0 \sigma}\left(\tau_{i}-\tau_{j}\right)
$$

and

$$
\left[F_{\sigma}^{\left\{s_{i}\right\}}\right]_{i j}=f_{\sigma}\left(s_{i}\right) \delta_{i j}
$$

respectively. Since the equality $K(s=1)=K(s=-1)$ holds for our choice of $\alpha_{\sigma}$ (Eq.(4)), we will simply denote them as $K$ hereafter.

\section{Monte Carlo sampling}

According to Eq. (12), the weight for the configuration $C_{n}$ is given by

$$
W\left(C_{n}\right)=\left(\frac{K d \tau}{2 \beta}\right)^{n} \times \prod_{\sigma} \operatorname{det} A_{\sigma}\left(C_{n}\right) .
$$

To guarantee the ergodicity, the addition and removal of the vertices with a random orientation of the auxiliary Ising spins $s_{i}= \pm 1$ at randomly-chosen imaginary times $\tau_{i} \in[0, \beta)$ are sufficient. To add a vertex, we randomly pick an imaginary time from the range $[0, \beta)$ and put there an auxiliary Ising spin with a randomly-chosen orientation, with a proposal probability of $P_{0}\left(C_{n} \rightarrow\right.$ $\left.C_{n+1}\right)=d \tau / 2 \beta$. To remove a vertex, we randomly choose one of the existing vertices, with the proposal probability $P_{0}\left(C_{n+1} \rightarrow C_{n}\right)=1 /(n+1)$. In the Metropolis algorithm, the acceptance ratio is

$$
P\left(C \rightarrow C^{\prime}\right)=\min \left(\frac{W\left(C^{\prime}\right) P_{0}\left(C^{\prime} \rightarrow C\right)}{W(C) P_{0}\left(C \rightarrow C^{\prime}\right)}, 1\right) .
$$

Applying this to the CT-INT, we obtain the acceptance ratios

$$
P\left(C_{n} \rightarrow C_{n+1}\right)=\min \left(\frac{K}{n+1} \prod_{\sigma} \frac{\operatorname{det} A_{\sigma}\left(C_{n+1}\right)}{\operatorname{det} A_{\sigma}\left(C_{n}\right)}, 1\right)
$$

for the addition of a vertex, and

$$
P\left(C_{n+1} \rightarrow C_{n}\right)=\min \left(\frac{n+1}{K} \prod_{\sigma} \frac{\operatorname{det} A_{\sigma}\left(C_{n}\right)}{\operatorname{det} A_{\sigma}\left(C_{n+1}\right)}, 1\right)
$$

for the removal of a vertex.

\section{Submatrix update}

In the conventional fast update scheme, the matrix $A_{\sigma}^{-1}$ is updated at each change of the auxiliary spins. Nukala et al $\stackrel{59}{\underline{59}}$ and subsequently Gull et al $\underline{\underline{60}}$ introduced a more efficient update algorithm, called submatrix update, to the Hirsch-Fye and the CT-AUX quantum Monte Carlo algorithms, respectively, where the matrix $A_{\sigma}^{-1}$ is updated at once after $k_{\max }$-time updates are done. The speed-up comes not from the reduction of the operation times, but from an efficient memory management by employing the matrix (submatrix) which is 
accommodated in a cache memory of the modern computer architectures, as is detailed in Ref 60. Here we introduce a similar submatrix update algorithm to the CT-INT, which is essential for implementing the multiorbital cDMFT calculation, described in Sec. IIB, in a practical computational time. We refer the readers to Refs. 59, 60 for a detailed derivation of Eqs. (24), (25), and (26) below, for which we avoid a repetition.

In the following we omit the spin index $\sigma$ for simplicity while the procedure described below has to be done for both spins $\sigma=\uparrow$ and $\downarrow$. We start from a configuration $C_{n}^{0}$. Suppose we know the corresponding matrix $A_{0}^{-1}\left(C_{n}^{0}\right)$ and that we propose insertions or removals of the auxiliary spins (vertices) for the next $k_{\max }$ times; let $k_{\max }^{\text {ins }}$ be the number of the insertions. We define an extended configuration $\tilde{C}_{n+k_{\max }^{\text {ins }}}^{0}$, which is comprised of the original configuration $C_{n}^{0}$ and the $k_{\max }^{\text {ins }}$ "noninteracting" vertices added at randomly-chosen imaginary times, i.e.,

$$
\begin{array}{r}
\tilde{C}_{n+k_{\max }^{\mathrm{ins}}}^{0}=\{\underbrace{\left(s_{1}^{0}, \tau_{1}^{0}\right), \cdots,\left(s_{n}^{0}, \tau_{n}^{0}\right)}_{C_{n}^{0}},\left(s_{n+1}^{0}=0, \tau_{n+1}^{0}\right), \\
\left.\cdots,\left(s_{n+k_{\max }^{\text {ins }}}^{\text {ins }}=0, \tau_{n+k_{\max }^{\text {ins }}}^{0}\right)\right\} .
\end{array}
$$

Then, we accordingly define an extended $\left(n+k_{\max }^{\text {ins }}\right) \times$ $\left(n+k_{\max }^{\text {ins }}\right)$ matrix $\tilde{A}_{0}^{-1}\left(\tilde{C}_{n+k_{\max }^{\text {ins }}}^{0}\right)$ by

$$
\tilde{A}_{0}^{-1}=\left(\begin{array}{cc}
A_{0} & 0 \\
B & 1
\end{array}\right)^{-1}=\left(\begin{array}{cc}
A_{0}^{-1} & 0 \\
-B A_{0}^{-1} & 1
\end{array}\right) .
$$

Here, $B$ is a $k_{\max }^{\text {ins }} \times n$ matrix with elements $B_{i j}=$ $-\tilde{\mathcal{G}}_{0}\left(\tau_{n+i}^{0}-\tau_{j}^{0}\right)\left(f\left(s_{j}^{0}\right)-1\right)$. Notice that the equality $\operatorname{det} A_{0}\left(C_{n}\right)=\operatorname{det} \tilde{A}_{0}\left(\tilde{C}_{n+k_{\text {max }}^{\text {ins }}}\right)$ holds, which is utilized in the calculation of the acceptance ratio described below.

With the extended matrix $\tilde{A}_{0}^{-1}$ and configuration $\tilde{C}_{n+k_{\max }^{\text {ins }}}^{0}$, the addition and the removal of the vertices can be done by just flipping the orientation of the auxiliary spins: The addition is expressed by changing an auxiliary spin $s$ from 0 to \pm 1 while the removal is expressed by the change from \pm 1 to 0 . Since the number of auxiliary spins (including those with zero value) is fixed during the spin-flip process, we abbreviate $\tilde{C}_{n+k_{\max }^{\text {ins }}}^{0}$ to $\tilde{C}^{0}$ below.

For later use, we denote the configuration after $k(<$ $\left.k_{\max }\right)$-th updates by $\tilde{C}^{k}$ and the auxiliary spins in $\tilde{C}^{k}$ by $\left\{s_{i}^{k}\right\}$. The positions of the flipped spins are denoted by $p_{j}\left(j=1,2, \cdots, l_{k} ; 1 \leq p_{j} \leq n+k_{\max }^{\text {ins }}\right)$ with $l_{k}$ being the number of the flipped spins. With these notations, we define an $l_{k} \times l_{k}$ matrix $\Gamma_{k}$ by

$$
\left[\Gamma_{k}\right]_{i j}=\left[\tilde{G}\left(\tilde{C}^{0}\right)\right]_{p_{i} p_{j}}-\delta_{i j} \frac{1+\gamma\left(s_{p_{i}}^{k}, s_{p_{i}}^{0}\right)}{\gamma\left(s_{p_{i}}^{k}, s_{p_{i}}^{0}\right)},
$$

with

$$
\gamma\left(s^{\prime}, s\right)=\frac{f\left(s^{\prime}\right)-f(s)}{f(s)} .
$$

The elements of the Green's function matrix $\left[\tilde{G}\left(\tilde{C}^{0}\right)\right]_{i j}$ can be efficiently calculated by using Eq. (A3) for $1 \leq$ $j \leq n$. For $n+1 \leq j \leq n+k_{\max }^{\text {ins }}$, we need to use Eq. (A4) to compute them since $s_{j}=0$. The matrix $\Gamma_{k}^{-1}$ is updated at each change of the auxiliary spins and is used to calculate the acceptance ratio. An efficient method to update $\Gamma_{k}^{-1}$ is elaborated in Ref. 60 and we do not repeat it here.

The acceptance ratios, Eqs. (43) and (44), can also be calculated easily from $\Gamma_{k}^{-1}$. Let us consider a $(k+1)$ th update at which the $p$-th spin is proposed to change from $s_{p}^{k}$ to $s_{p}^{\prime k}$ and the configuration moves from $\tilde{C}^{k}$ to $\tilde{C}^{\prime k}$. When $p \neq p_{j}$ for $j=1,2, \cdots l_{k}$, the determinant ratio is given by

$$
\frac{\operatorname{det} \tilde{A}_{k}^{\prime}}{\operatorname{det} \tilde{A}_{k}}=-\gamma\left(s_{p}^{\prime k}, s_{p}^{k}\right) \frac{\operatorname{det} \Gamma_{k}^{\prime}}{\operatorname{det} \Gamma_{k}},
$$

where $\Gamma_{k}^{\prime}$ is an $\left(l_{k}+1\right) \times\left(l_{k}+1\right)$ matrix whose elements of the $\left(l_{k}+1\right)$-th row and column are calculated from Eq. (22) with $p_{l_{k}+1}=p$. Otherwise, $p$ coincides with one of $\left\{p_{j}\right\}\left(j=1,2, \cdots l_{k}\right)$, i.e., a previously inserted vertex is proposed to be removed. In this case, the $p$-th spin has already been changed from $s_{p}^{0}=0$ to $s_{p}^{k}= \pm 1$, and therefore $s_{p}^{\prime k}=0=s_{p}^{0}$. Then the determinant ratio is given by

$$
\frac{\operatorname{det} \tilde{A}_{k}^{\prime}}{\operatorname{det} \tilde{A}_{k}}=-\frac{1}{\gamma\left(s_{p}^{k}, 0\right)} \frac{\operatorname{det} \Gamma_{k}^{\prime}}{\operatorname{det} \Gamma_{k}} .
$$

Here $\Gamma_{k}^{\prime}$ is an $\left(l_{k}-1\right) \times\left(l_{k}-1\right)$ matrix in which a column and a row corresponding to $p$-th spin are removed from $\Gamma_{k}$.

If the proposal is accepted, the proposed configuration $\tilde{C}^{\prime k}$ becomes the new configuration $\tilde{C}^{k+1}$, and accordingly, the size of the $\Gamma$ matrix increases or decreases. Otherwise, the configuration and the $\Gamma$ matrix are unchanged. Then, we move to the $(k+2)$-th update. We repeat this procedure up to $k_{\max }$ times.

After $k_{\max }$-th update, we recompute the $A^{-1}$ matrix. To this end, we use the identity $\underline{59,60}$

$$
\left[\tilde{A}_{k_{\max }}^{-1}\right]_{i j}=\frac{\left[\tilde{A}_{0}^{-1}\right]_{i j}-\left[\tilde{G}\left(\tilde{C}^{0}\right)\right]_{i p_{k}}\left[\Gamma_{k_{\max }}^{-1}\right]_{p_{k} p_{l}}\left[\tilde{A}_{0}^{-1}\right]_{p_{l} j}}{1+\gamma\left(s_{i}^{k_{\max }}, s_{i}^{0}\right)} .
$$

We then delete the "noninteracting" auxiliary spins from $\tilde{A}_{k_{\max }}^{-1}$ by removing the corresponding rows and columns and obtain a new $A^{-1}$ matrix, which gives the starting point for the next $k_{\max }$-times updates.

\section{B. Multi-orbital case}

\section{Extension to the multi-orbital systems with the} conventional single-vertex update

We now extend the above algorithm to the multiorbital case. The action of the multi-orbital impurity 
problem is given by

$$
S_{\mathrm{imp}}=S_{0}+S_{\mathrm{int}},
$$

where

$$
S_{0}=-\int_{0}^{\beta} d \tau \int_{0}^{\beta} d \tau^{\prime} \sum_{i j, \sigma}\left[\mathcal{G}_{0 \sigma}^{-1}\left(\tau-\tau^{\prime}\right)\right]_{i j} \hat{c}_{i \sigma}^{\dagger}(\tau) \hat{c}_{j \sigma}\left(\tau^{\prime}\right)
$$

and

$$
\begin{aligned}
S_{\mathrm{int}}=\int_{0}^{\beta} d \tau & {\left[\sum_{i} U \hat{n}_{i \uparrow}(\tau) \hat{n}_{i \downarrow}(\tau)+\sum_{i<j, \sigma} U^{\prime} \hat{n}_{i \sigma}(\tau) \hat{n}_{j \bar{\sigma}}(\tau)\right.} \\
& +\sum_{i<j, \sigma}\left(U^{\prime}-J_{\mathrm{H}}\right) \hat{n}_{i \sigma}(\tau) \hat{n}_{j \sigma}(\tau) \\
& +\sum_{i \neq j} J_{\mathrm{H}} \hat{c}_{i \uparrow}^{\dagger}(\tau) \hat{c}_{j \uparrow}(\tau) \hat{c}_{j \downarrow}^{\dagger}(\tau) \hat{c}_{i \downarrow}(\tau) \\
& \left.+\sum_{i \neq j} J_{\mathrm{H}} \hat{c}_{i \uparrow}^{\dagger}(\tau) \hat{c}_{j \uparrow}(\tau) \hat{c}_{i \downarrow}^{\dagger}(\tau) \hat{c}_{j \downarrow}(\tau)\right]
\end{aligned}
$$

Here, the Weiss function $\mathcal{G}_{0 \sigma}^{-1}\left(\tau-\tau^{\prime}\right)$ is a matrix with respect to the orbital $i$ and $j$. $U, U^{\prime}$, and $J_{\mathrm{H}}$ are the intra-orbital Coulomb interaction, inter-orbital Coulomb interaction, and Hund's coupling, respectively. $\hat{c}_{i \sigma}^{\dagger}\left(\hat{c}_{i \sigma}\right)$ is a Grassmann variable representing the creation (annihilation) of the impurity electron with the orbital $i$ and the spin $\sigma$, and $\hat{n}_{i \sigma}=\hat{c}_{i \sigma}^{\dagger} \hat{c}_{i \sigma}$.

As in the single-orbital case, we introduce additional parameters. We employ ${ }^{62}$

$$
\begin{aligned}
& \alpha_{1 \uparrow}(s)=1 / 2+s \delta_{1} \\
& \alpha_{1 \downarrow}(s)=1 / 2-s \delta_{1}
\end{aligned}
$$

with $s= \pm 1$ and $\delta_{1}=1 / 2+0^{+}$, and

$$
\begin{aligned}
& \alpha_{2 \uparrow}(s)=+s \delta_{2} \\
& \alpha_{2 \downarrow}(s)=-s \delta_{2}
\end{aligned}
$$

with a small positive real number $\delta_{2}$. Then we rewrite the non-interacting part of the action as

$$
S_{0}=-\int_{0}^{\beta} d \tau \int_{0}^{\beta} d \tau^{\prime} \sum_{i j, \sigma}\left[\tilde{\mathcal{G}}_{0 \sigma}^{-1}\left(\tau-\tau^{\prime}\right)\right]_{i j} \hat{c}_{i \sigma}^{\dagger}(\tau) \hat{c}_{j \sigma}\left(\tau^{\prime}\right)
$$

where $\tilde{\mathcal{G}}_{0 \sigma}$ is the local noninteracting Green's function defined at a modified chemical potential $\tilde{\mu}=\mu-U / 2-$ $N_{\text {orb }}\left(2 U^{\prime}-J_{\mathrm{H}}\right) / 2$ with $N_{\text {orb }}$ being the number of the orbitals. The interaction part of the action is

$$
\begin{aligned}
S_{\mathrm{int}} & =\int_{0}^{\beta} d \tau \sum_{s= \pm 1}\left[\sum_{i} \frac{U}{2}\left[\hat{n}_{i \uparrow}(\tau)-\alpha_{1 \uparrow}(s)\right]\left[\hat{n}_{i \downarrow}(\tau)-\alpha_{1 \downarrow}(s)\right]+\sum_{i<j, \sigma} \frac{U^{\prime}}{2}\left[\hat{n}_{i \sigma}(\tau)-\alpha_{1 \sigma}(s)\right]\left[\hat{n}_{j \bar{\sigma}}(\tau)-\alpha_{1 \bar{\sigma}}(s)\right]\right. \\
& +\sum_{i<j, \sigma} \frac{U^{\prime}-J_{\mathrm{H}}}{2}\left[\hat{n}_{i \sigma}(\tau)-\alpha_{1 \sigma}(s)\right]\left[\hat{n}_{j \sigma}(\tau)-\alpha_{1 \sigma}(s)\right]+\sum_{i \neq j} \frac{J_{\mathrm{H}}}{2}\left[\hat{c}_{i \uparrow}^{\dagger}(\tau) \hat{c}_{j \uparrow}(\tau)-\alpha_{2 \uparrow}(s)\right]\left[\hat{c}_{j \downarrow}^{\dagger}(\tau) \hat{c}_{i \downarrow}(\tau)-\alpha_{2 \downarrow}(s)\right] \\
& \left.+\sum_{i \neq j} \frac{J_{\mathrm{H}}}{2}\left[\hat{c}_{i \uparrow}^{\dagger}(\tau) \hat{c}_{j \uparrow}(\tau)-\alpha_{2 \uparrow}(s)\right]\left[\hat{c}_{i \downarrow}^{\dagger}(\tau) \hat{c}_{j \downarrow}(\tau)-\alpha_{2 \downarrow}(s)\right]\right]
\end{aligned}
$$

Thanks to the $\alpha_{1}$ terms, we can avoid the negative signs coming from the density-density interactions as in the Hirsch-Fye and CT-AUX algorithms ${ }^{62}$ Without them, negative signs appear since the $V$ matrix corresponding to the density-density-type vertex in Eq. (B2) obtains matrix elements with negative values 61 On the other hand, the number of negative signs increases with $\delta_{2}$. However, as far as the off-diagonal parts of the Weiss function vanish (i.e., $\left[\tilde{\mathcal{G}}_{0 \sigma}\right]_{i j}=0$ for $i \neq j$ ), we need a non-zero $\delta_{2}$ value to satisfy the ergodicity. In the twoorbital case, we can incorporate the last two terms in Eq. (33) more efficiently, as we shall discuss in Sec. IIB2

If we neglect the spin-flip and pair-hopping terms, which correspond to the last two terms in Eq. (33), we only have the density-density type interactions and the symmetry of the spin lowers from $\mathrm{SU}(2)$ to $Z_{2}$. This mitigates the sign problem considerably and hence often employed in literature though the neglect has no physical ground $53-66$ Hereafter, we call the Hamiltonian with the spin-flip and pair-hopping terms as SU(2)-symmetric Hamiltonian, and the Hamiltonian without them as $Z_{2^{-}}$ symmetric Hamiltonian.

In the multi-orbital case, we define a configuration as

$$
C_{n}=\left\{\left(\kappa_{1}, s_{1}, \tau_{1}\right), \cdots,\left(\kappa_{n}, s_{n}, \tau_{n}\right)\right\}
$$

where we introduce the index $\kappa$ for the type of the interaction. We also need to generalize the $f$ and $K$ functions: In the case where $\kappa$ designates a density-density interac- 
tion, we define $f$ as

$$
f_{\kappa \sigma}(s)=\left\{\begin{array}{ll}
\frac{\alpha_{1 \sigma}(s)}{\alpha_{1 \sigma}(s)-1} & s= \pm 1 \\
1 & s=0
\end{array},\right.
$$

otherwise, it is defined as

$$
f_{\kappa \sigma}(s)=\left\{\begin{array}{ll}
\frac{\alpha_{2 \sigma}(s)}{\alpha_{2 \sigma}(s)-1} & s= \pm 1 \\
1 & s=0
\end{array} .\right.
$$

Then the $K$ function is defined by

$$
K_{\kappa}(s)=\frac{-\beta V_{\kappa}}{\left(f_{\kappa \uparrow}(s)-1\right)\left(f_{\kappa \downarrow}(s)-1\right)},
$$

for $s= \pm 1$ with $V_{\kappa}=U, U^{\prime}, U^{\prime}-J_{\mathrm{H}}$, or $J_{\mathrm{H}}$.

With these functions, the partition function for the multi-orbital impurity problem is written in the form

$$
\begin{aligned}
\frac{Z}{Z_{0}}= & \sum_{n=0}^{\infty} \int_{0}^{\beta} \sum_{\kappa_{1}} \sum_{s_{1}= \pm 1} \cdots \int_{0}^{\tau_{n-1}} \sum_{\kappa_{n}} \sum_{s_{n}= \pm 1} \\
& {\left[\prod_{i=1}^{n} \frac{K_{\kappa_{i}}\left(s_{i}\right) d \tau_{i}}{2 \beta} \times \prod_{\sigma} \operatorname{det} A_{\sigma}\left(C_{n}\right)\right] . }
\end{aligned}
$$

The $A$ matrix has a similar form as that in Eq. (13), but now we have an additional orbital indices for the $G_{0}$ matrix and $\kappa$ index for the $F$ matrix. When the interaction between the same spin (the third term in Eq. (33)) is inserted, the size of the $A$ matrix for that spin increases by two, while no increase for the opposite spin. Therefore, the size of the $A$ matrix does not necessarily agree with the number of the interaction vertices $n$, while (size of $\left.A_{\uparrow}\right)+\left(\right.$ size of $\left.A_{\downarrow}\right)=2 n$ holds.

Now the application of the submatrix update to the multi-orbital case is straightforward. We only comment on several important differences from the single-orbital one. (i) We need to modify the definition of the $\gamma$ function to have $\kappa$ index. (ii) As in the $A$ matrix, the sizes of the $\Gamma_{\uparrow}$ and $\Gamma_{\downarrow}$ matrices do not necessarily agree. (iii) If the update is related to the interaction between the same spin, we need to enlarge or shrink the $\Gamma$ matrix by two rows and two columns only for the relevant spin components.

\section{Efficient sampling scheme for the spin-flip and pair-hopping terms: Double-vertex update}

Here, we show, in the two-orbital Hubbard model without a hybridization between the orbitals, that the spin-flip and pair-hopping interactions can be treated efficiently by incorporating the double-vertex insertion and removal processes, on top of the standard singlevertex updates for the density-density-type interactions.
The double-vertex update allows the spin-flip and pairhopping interactions to appear only at even perturbation orders, eliminating unphysical odd-order terms, and thus suppresses the negative sign problem coming from these interactions. In particular, in the single-site DMFT, we find that the negative signs are absent.

In order to clue in our idea, let us look into Eq. (33) again. Suppose that there is no hybridization between the two orbitals, that is, $\left[\tilde{\mathcal{G}}_{0 \sigma}\right]_{12}=\left[\tilde{\mathcal{G}}_{0 \sigma}\right]_{21}=0$. Then we can easily see that, without $\delta_{2}$, the thermal average of the products of the Grassmann variables, $\left\langle V\left[\hat{c}_{1 \sigma}^{\dagger}, \hat{c}_{2 \sigma}^{\dagger}, \hat{c}_{1 \sigma}, \hat{c}_{2 \sigma}\right]\right\rangle_{0}$, can be finite only when the equality (number of $\hat{c}_{i \sigma}^{\dagger}$ in $V$ ) $=$ (number of $\hat{c}_{i \sigma}$ in $V$ ) holds for each $i=1,2$ and $\sigma=\uparrow, \downarrow$. This condition is always satisfied when only the density-type vertices come in. However, a single non-density-type vertex (spin-flip or pairhopping) does not meet this condition, and therefore it must always appear in pair with another corresponding non-density-type vertex in order to have a finite contribution. Nevertheless, when $\delta_{2}$ is non-zero, a configuration with the odd number of the non-density-type vertices can have a finite weight because of the constant $\alpha_{2 \sigma}$. While the presence of these odd-order terms is artificial, they are necessary to keep the ergodicity within the singlevertex update processes since in this case the number of the non-density-type vertices cannot be changed without passing through the odd-order terms.

The above consideration motivates us to introduce double-vertex insertion or removal processes for the spinflip and pair-hopping terms, where we insert or remove two non-density-type vertices at different imaginary times simultaneously. With the double-vertex update processes we can sample over only the even-order terms with respect to the non-density-density interactions so that we can avoid the negative signs coming from the artificial odd-order terms. The idea can apply to both the conventional and submatrix update algorithms. While the double-vertex update dispenses with the additional parameter $\delta_{2}$ in the conventional fast update scheme, in order to apply the submatrix update, we introduce another type of parameters,

$$
\begin{aligned}
& \alpha_{3 \uparrow}(s)=+s \delta_{3} \\
& \alpha_{3 \downarrow}(s)=-s \delta_{3}
\end{aligned}
$$

and

$$
\begin{aligned}
& \alpha_{4 \uparrow}(s)=+s \delta_{3} \\
& \alpha_{4 \downarrow}(s)=+s \delta_{3}
\end{aligned}
$$

with $s= \pm 1$ and a positive real number $\delta_{3}$. These parameters are needed to avoid the divergence of $\gamma$ function in Eq. (23). We rewrite the action for the spin-flip and pair-hopping part as 


$$
\begin{aligned}
& S_{\text {int }}^{\text {non-dens. }}=\int_{0}^{\beta} d \tau \sum_{s= \pm 1}[\underbrace{\sum_{l=3,4} \frac{J_{\mathrm{H}}}{4}\left[\hat{c}_{1 \uparrow}^{\dagger}(\tau) \hat{c}_{2 \uparrow}(\tau)-\alpha_{l \uparrow}(s)\right]\left[\hat{c}_{2 \downarrow}^{\dagger}(\tau) \hat{c}_{1 \downarrow}(\tau)-\alpha_{l \downarrow}(s)\right]}_{\kappa=7} \\
& +\underbrace{\sum_{l=3,4} \frac{J_{\mathrm{H}}}{4}\left[\hat{c}_{2 \uparrow}^{\dagger}(\tau) \hat{c}_{1 \uparrow}(\tau)-\alpha_{l \uparrow}(s)\right]\left[\hat{c}_{1 \downarrow}^{\dagger}(\tau) \hat{c}_{2 \downarrow}(\tau)-\alpha_{l \downarrow}(s)\right]}_{\kappa=8} \\
& +\underbrace{\sum_{l=3,4} \frac{J_{\mathrm{H}}}{4}\left[\hat{c}_{1 \uparrow}^{\dagger}(\tau) \hat{c}_{2 \uparrow}(\tau)-\alpha_{l \uparrow}(s)\right]\left[\hat{c}_{1 \downarrow}^{\dagger}(\tau) \hat{c}_{2 \downarrow}(\tau)-\alpha_{l \downarrow}(s)\right]}_{\kappa=9} \\
& +\underbrace{\left.\sum_{l=3,4} \frac{J_{\mathrm{H}}}{4}\left[\hat{c}_{2 \uparrow}^{\dagger}(\tau) \hat{c}_{1 \uparrow}(\tau)-\alpha_{l \uparrow}(s)\right]\left[\hat{c}_{2 \downarrow}^{\dagger}(\tau) \hat{c}_{1 \downarrow}(\tau)-\alpha_{l \downarrow}(s)\right]\right]}_{\kappa=10} .
\end{aligned}
$$

The idea behind this form of the additional parameters $\alpha_{3 \sigma}$ and $\alpha_{4 \sigma}$ is to eliminate the weight of the odd-order terms, as one can easily verify it by seeing that the sum over $s$ and $l$ for the each term on the right hand side of Eq. (41) reproduces the original action for the spin-flip and pair-hopping terms without the additional constant. We use the same $S_{0}$ and the density-density part of $S_{\text {int }}$ as those in Eqs. (32) and (33), where we assign $\kappa=1-6$ to the density-density interactions in $S_{\text {int }}$. In the update, the $\kappa=7$ vertex has to be paired with $\kappa=8$ vertex. In the same way, the $\kappa=9$ vertex has to be paired with $\kappa=10$ vertex. In principle, $\delta_{3}$ is arbitrary as far as it is nonzero, however, a small value is preferable because in the $\delta_{3} \rightarrow 0$ limit, we can prove mathematically that the negative signs are absent (see Appendix $\mathrm{B}$ ). We set $\delta_{3}$ to be $\sim 10^{-4}$, and with this small value, we do not encounter the negative signs as will be shown in Sec. IIA A large value of $\delta_{3}$ will increase the matrix size and produce the negative signs.

$f$ function for the non-density-type vertices is modified to

$$
f_{\kappa \sigma}(l, s)= \begin{cases}\frac{\alpha_{l \sigma}(s)}{\alpha_{l \sigma}(s)-1} & s= \pm 1 \\ 1 & s=0\end{cases}
$$

for $\kappa=7-10$ and $l=3,4$. Correspondingly we define $V_{\kappa}=J_{\mathrm{H}} / 2$, with which the partition function is given in the same form as Eq. (38).

At the insertion update, we propose the double vertex with a probability $R$, and the single vertex with $1-R$. When the double-vertex update is selected, we randomly choose either pair of $(7,8)$ or $(9,10)$. Then, we pick two imaginary times from the range $[0, \beta)$ and assign the $l$ value $(l=3,4)$ and auxiliary spin orientations $(s= \pm 1)$ for each vertex in the pair. Eventually a proposal ratio for inserting a certain pair of the non-density-type vertices is $R / 32 \times(d \tau / \beta)^{2}$. As for the removal update, we first pick randomly one of the existing vertices. If the chosen vertex is of density-density type, we propose the single-vertex removal. Otherwise, we propose the doublevertex removal: If the type of the chosen vertex is 7 , for example, we additionally choose one vertex from the existing $\kappa=8$ vertices with a probability $1 / m_{\kappa=8}$ with $m_{\kappa=8}$ being the number of $\kappa=8$ vertices in the configuration. Then a proposal ratio for removing a $(7,8)$ pair is $\frac{2}{n m_{\kappa}-7}$, where $n$ is the number of existing vertices of all kinds. The factor of 2 in the numerator comes from the sum of probability for the case where the first-chosen vertex is of $\kappa=7$ and $\kappa=8$. Note that $m_{\kappa=7}=m_{\kappa=8}$ and $m_{\kappa=9}=m_{\kappa=10}$ always hold during the simulation. With $X=\frac{16 K_{\kappa=7}^{2}}{R(n+2)\left(m_{\kappa=7}+1\right)}$, the acceptance ratio concerning (7,9)-pair vertices is

$$
P\left(C_{n} \rightarrow C_{n+2}\right)=\min \left(X \prod_{\sigma} \frac{\operatorname{det} A_{\sigma}\left(C_{n+2}\right)}{\operatorname{det} A_{\sigma}\left(C_{n}\right)}, 1\right)
$$

for the addition process and

$$
P\left(C_{n+2} \rightarrow C_{n}\right)=\min \left(\frac{1}{X} \prod_{\sigma} \frac{\operatorname{det} A_{\sigma}\left(C_{n}\right)}{\operatorname{det} A_{\sigma}\left(C_{n+2}\right)}, 1\right)
$$

for the removal process. The acceptance ratios for the insertion and the removal of the other vertex pairs are calculated in the same way.

Suppose a pair of the auxiliary spins, $\left(s_{p}^{k}, s_{q}^{k}\right)$, is proposed to change to $\left(s_{p}^{\prime k}, s_{q}^{\prime k}\right)$ by the double-vertex update. As far as $p$-th and $q$-th spins have not been changed in the previous $(k-1)$ steps, the change of the type $(0,0) \rightarrow( \pm 1, \pm 1)$ (insertion) or $( \pm 1, \pm 1) \rightarrow(0,0)$ (removal) will enlarge the $\Gamma$ matrix by two rows and two columns if accepted. If both the $p$-th and $q$-th spins have already been flipped from 0 to \pm 1 (insertion), the change at the $k$-th step is of the type $( \pm 1, \pm 1) \rightarrow(0,0)$ (removal) 
and the $\Gamma$ matrix will shrink by two rows and two columns if accepted, since both the $p$-th and $q$-th spins return to the original orientations $(s=0)$. Otherwise, one of the two spins, say the $p$-th spin, has been changed in the previous $(k-1)$ steps while the other (the $q$-th spin) has not. In this case, the change is of the type $( \pm 1, \pm 1) \rightarrow(0,0)$ (removal) and in the $\Gamma$ matrix one row and one column will be added for the $q$-th spin while one row and one column concerning the $p$-th spin will be removed if accepted.

Finally, we comment on the three-orbital case. Suppose that there is no hybridization among the orbitals. In this case, on top of the double-vertex update, we will need the triple-vertex update, where, three spin-flip interactions involving the orbital pairs $(1,2),(2,3)$, and $(3,1)$, for example, are inserted or removed.

\section{Multi-orbital and multi-site case}

It is straightforward to extend the above-described algorithm, both the single-vertex and double-vertex updates, to the multi-orbital and multi-site impurity problem. We only need to define a "generalized orbital" which specifies the site and the orbital simultaneously. With these "generalized orbitals", we can employ the same method described in Sec. IIB. For example, when we consider two-orbital and two-site case, the "generalized orbital' runs from 1 to 4: "Generalized orbital" 1, 2, 3, and 4 denote the orbital 1 at the site 1 , the orbital 2 at the site 1 , the orbital 1 at the site 2 , and the orbital 2 at the site 2 , respectively. The Weiss function becomes a matrix with respect to the "generalized orbitals" and includes the off-site processes, e.g., $\left[\tilde{\mathcal{G}}_{0 \sigma}^{-1}\left(\tau-\tau^{\prime}\right)\right]_{13}$. It also should be noted that, for the multi-orbital Hubbard model, the interactions exist only within the "orbital" 1 and the "orbital" 2, and within the "orbital" 3 and the "orbital" 4.

\section{RESULTS}

Here, we show numerical results for the 2D two-orbital Hubbard model. We consider two degenerate orbitals on a square lattice with only the nearest neighbor intraorbital hopping $t$, which is used as the unit of energy, i.e., $t=1$. The electron density is set to be half filling. We implement the cellular DMFT with a four-site cluster, in which the impurity problem has $2 \times 4=8$ degrees of freedom in total, and compare the results with those of the single-site DMFT to elucidate the effect of shortrange spatial correlations.

The impurity problem is solved by the CT-INT method described in the previous section, where the Legendre orthogonal polynomials expansion of the imaginary-time Green's function is employed as a "noise filter" 67 We restrict ourselves to the paramagnetic and para-orbital solution to clarify the nature of the Mott metal-insulator transition. We explicitly treat the spin-flip and pairhopping terms (the SU(2)-symmetric Hamiltonian) and compare the result with that of the $Z_{2}$-symmetric Hamiltonian.

For the $\mathrm{SU}(2)$-symmetric Hamiltonian at $T / t=0.05$ and $U / t=5.4$, where the calculation is severest in the present study, the average expansion order of the interaction vertices reaches $\sim 740$ and we take 1,536,000 QMC steps to solve the impurity problem. In this case, it takes about one hour with 512-core parallelization (clock frequency: $2.90 \mathrm{GHz}$ ) to perform one self-consistent loop.

\section{A. Comparison between single-vertex and double-vertex updates}

Before going to the physical results for the 2D twoorbital Hubbard model, we demonstrate how much the negative signs are reduced by employing the doublevertex update for the spin-flip and pair-hopping terms. The calculation is performed at $U=6 t, U^{\prime}=3 t$ and $J_{\mathrm{H}}=1.5 t$. Fig. 1(a) shows the single-site DMFT results of the average sign for the SU(2)-symmetric Hamiltonian at several temperatures. As can be seen, the double-vertex update always gives the average sign of 1, eliminating the negative signs completely. On the other hand, the single-vertex update suffers from the negative signs, which become severer as the temperature decreases. Since the slope in Fig. 1(a) is more modest for the smaller value of $\delta_{2}$, one might think that if we further decrease $\delta_{2}$, we can get rid of the sign problem. However, if $\delta_{2}$ is too small, the calculation becomes unstable, as seen in Fig. 11(b): The result with $\delta_{2}=10^{-3}$ strongly fluctuates around the right value (red and blue curves) $\sim 0.08$, and for $\delta_{2}=10^{-4}$ even the average value of the solution deviates from the right one. The result with $\delta_{2}=10^{-4}$ is rather close to the result with the $Z_{2}$-symmetric Hamiltonian. This is reasonable because the reduction of $\delta_{2}$ suppresses the flip to the odd-order non-density-type terms: Since we start from the noninteracting limit (0th order), the smaller $\delta_{2}$ lessens the chance to have a finite-order non-density-type terms, resulting in a double-occupancy value similar to the $Z_{2}$ symmetric one. Therefore, if we want an accurate and stable result with the single-vertex update, we need to use a substantial value for $\delta_{2}$, which inevitably causes negative signs. On the other hand, in the double-vertex update, the accuracy does not essentially depend on the choice of $\delta_{3}$, and as far as we use a small value for $\delta_{3}$, we see that the average sign is always one. The computational time highly depends on the average sign: If the average sign is 0.5 , we need a twice larger calculation to get the same effective sampling numbers as that of (average sign) $=1$ case. Therefore, the double-vertex update saves the computational time significantly. 

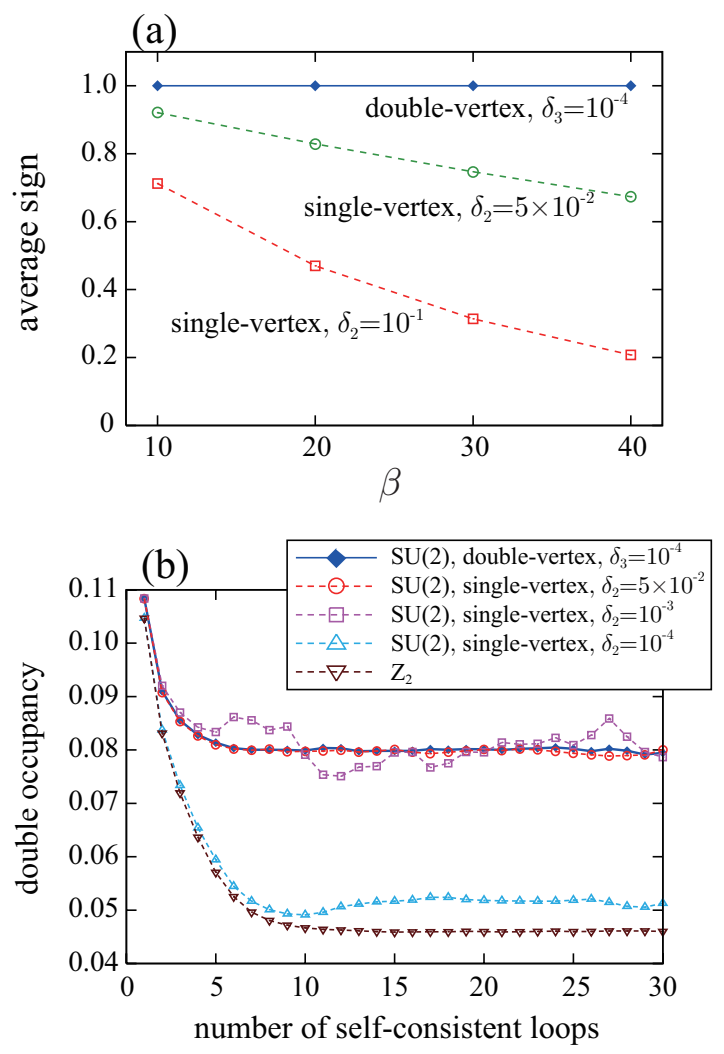

FIG. 1: (Color online) (a) The average sign for the SU(2)-symmetric Hamiltonian obtained within the single-site DMFT. Filled (open) symbols show the results with the double-vertex (single-vertex) update for the spin-flip and pairhopping terms. (b) The double occupancy for each orbital for the $\mathrm{SU}(2)$-symmetric Hamiltonian at $\beta=20$ as a function of the number of the self-consistent loops, where we employ the single-site DMFT. For comparison, we also show the results for the $Z_{2}$-symmetric Hamiltonian. We start the selfconsistent loop from the non-interacting limit, and we fully update the Weiss function at each loop. In the QMC simulation, 320,000 measurements are done. The calculation is performed with $U=6 t$, and $U^{\prime}=3 t$ and $J_{\mathrm{H}}=1.5 t$ both for the panels (a) and (b).

\section{B. Phase diagram}

Figures 2(a) and 2(b) show the phase diagrams with respect to the temperature $T$ and the interaction $U$ for the $\mathrm{SU}(2)$-symmetric Hamiltonian and the $Z_{2}$-symmetric one, respectively, where the ratio between Hund's coupling $J_{\mathrm{H}}$ and the Hubbard interaction $U$ is set to be $J_{\mathrm{H}} / U=1 / 6$, and $U^{\prime}=U-2 J_{\mathrm{H}}$. The ratio $J_{\mathrm{H}} / U=1 / 6$ is close to that of the transition metal oxides, $\stackrel{68,69}{\text { typi- }}$ cal multi-orbital strongly correlated materials. The color contour plot indicates the double occupancy obtained by the solution approached from the metallic side. The raw data of the double occupancy are shown in Fig. 3. The transition from a metallic state to the Mott insulating state can be identified by the abrupt change in the dou-
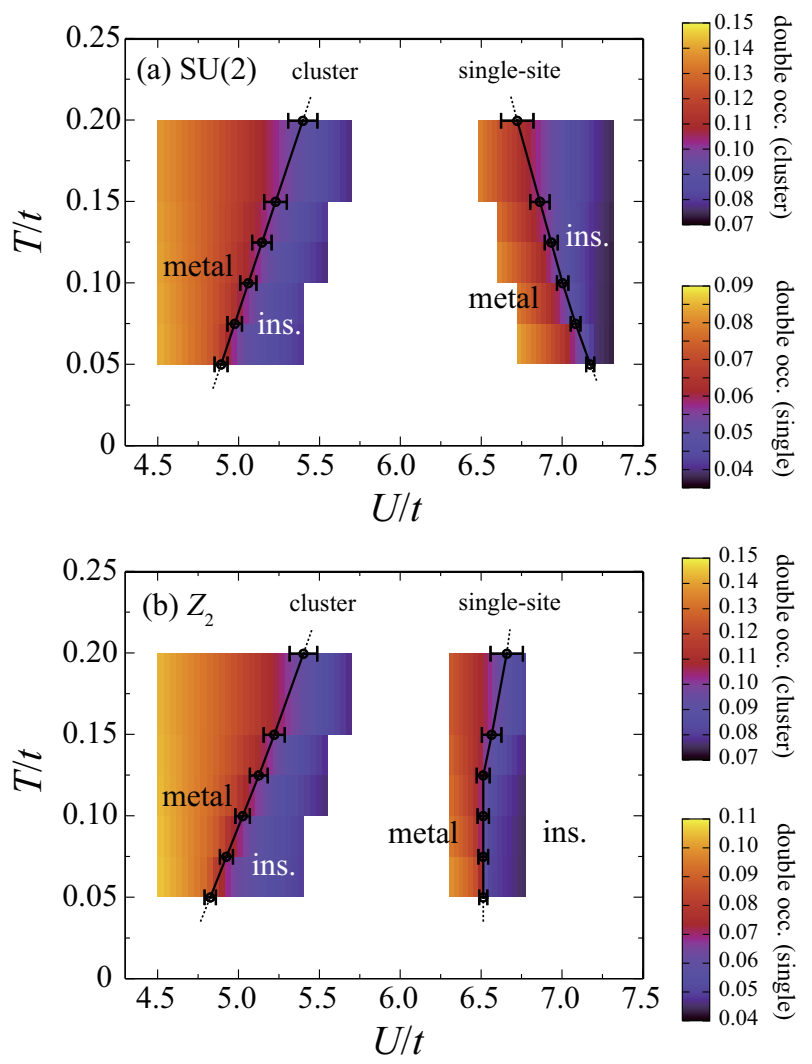

FIG. 2: (Color online) Phase diagrams obtained by the cDMFT and single-site DMFT for (a) the $\mathrm{SU}(2)$-symmetric Hamiltonian and (b) the $Z_{2}$-symmetric Hamiltonian with $J_{\mathrm{H}} / U=1 / 6$ and $U^{\prime}=U-2 J_{\mathrm{H}}$. Color contour plots show the double occupancy for each orbital, where the data between the calculated points are estimated by a linear interpolation. The solid lines show the phase boundary at which the metallic solution becomes unstable, or the crossover line determined by the maximal point of the first derivative of the double occupancy as a function of $U$. Within the present resolution, we could not determine the critical end point precisely.

ble occupancy. As the temperature increases, the change gets smoother and goes on to a crossover-like behavior, where we determine the crossover line by the maximal point of the first derivative of the double occupancy curves as a function of $U$. In Figs. 2(a) and2(b), we show thus-estimated phase boundary $U_{c_{2}}$ or the crossover line of the Mott metal-insulator transition obtained by the single-site and cellular DMFTs.

First, we comment on the single-site DMFT results. In the $\mathrm{SU}(2)$-symmetric case, the critical interaction strength increases as the temperature decreases, which reflects the fact that the paramagnetic insulating state has a larger entropy than the metallic state, as in the single-orbital case. On the other hand, $U_{c_{2}}$ for $Z_{2^{-}}$ symmetric Hamiltonian is almost unchanged with respect to the temperature while in the crossover region $(T \gtrsim 0.12 t)$, the crossover line shifts to a larger $U$ as the temperature increases. The different slopes between 

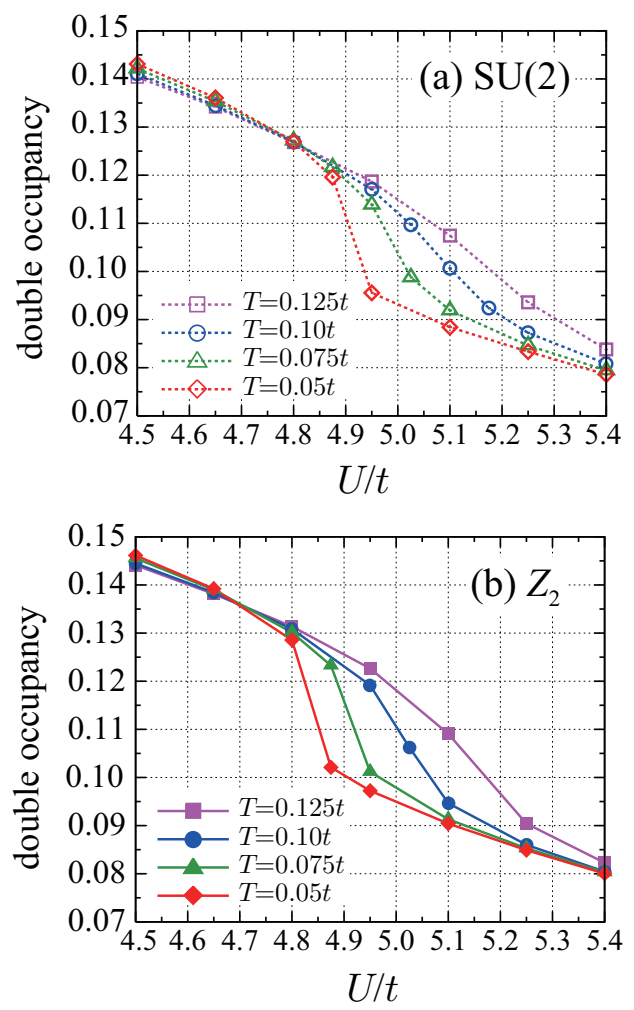

FIG. 3: (Color online) The cDMFT results for the double occupancy for each orbital for (a) the SU(2)-symmetric Hamiltonian and (b) the $Z_{2}$-symmetric Hamiltonian with $J_{\mathrm{H}} / U=1 / 6$ and $U^{\prime}=U-2 J_{\mathrm{H}}$. The lines are guides to the eye. The sizes of the error bars are slightly small compared to the sizes of symbols.

$\mathrm{SU}(2)$ and $Z_{2}$ come from their different ground-state degeneracy in the atomic limit where each orbital has one electron with a spin oriented to the same direction $(S=1)$. In the $\mathrm{SU}(2)$ case the ground state is triply degenerate $\left(S_{z}=1,0,-1\right)$ while in the $Z_{2}$ case it is doubly degenerate $\left(S_{z}= \pm 1\right)$. Hence, the insulating state in the $\mathrm{SU}(2)$-symmetric Hamiltonian has a larger entropy than that in the $Z_{2}$-symmetric Hamiltonian, accounting for the tendency to have a negative slope of the phase boundary in the SU(2) case. Furthermore, in the metallic region for the $Z_{2}$-symmetric Hamiltonian, since the system is locked into the states with $S_{z}= \pm 1$ due to a strong Hund's coupling, the Kondo screening is inefficient, $\stackrel{70}{\underline{a}}$ while it works in the $\mathrm{SU}(2)$-symmetric Hamiltonian as well as in the single-orbital one. Therefore, the metallic state in the multi-orbital $Z_{2}$ case has a larger entropy than that in the multi-orbital SU(2) and singleorbital cases. Since in the atomic limit both the singleorbital and multi-orbital $Z_{2}$ Hamiltonians have the same ground-state degeneracy of two, which would give a similar entropy in the insulating region, the above-mentioned difference in the metallic state would explain the positive slope in the $Z_{2}$ case. Notice also that the $Z_{2}$-symmetric
Hamiltonian significantly overestimates the tendency toward the insulator compared to the $\mathrm{SU}(2)$-symmetric one.

We now turn to the cellular DMFT results. Due to the short-range spatial correlations, the critical interaction strength for the Mott transition considerably decreases. It is interesting to note that the difference in the critical interaction strength between $\mathrm{SU}(2)$ - and $Z_{2^{-}}$ symmetric Hamiltonians is much smaller than that in the single-site DMFT. By comparing Figs. 3(a) and 3(b), we find that the $Z_{2}$-symmetric Hamiltonian overestimates the tendency toward the insulator while the difference of the critical interaction is less than 0.1t. In contrast to the single-site DMFT results, the slopes of the phase boundary in Fig. 2 are also similar between the SU(2)and $Z_{2}$-symmetric Hamiltonians: The critical interaction strength decreases as the temperature decreases in both cases. In the $\mathrm{SU}(2)$-symmetric Hamiltonian, in analogy with the single-orbital case, $\stackrel{71}{=}$ this would be attributed to the entropy reduction of the insulating phase by the formation of the inter-site singlets within the cluster. In the $Z_{2}$-symmetric case, the Ising-type antiferromagnetic spin alignment would be favored in the cluster and thus the insulating phase has a smaller entropy than that in the single-site DMFT. To confirm these scenarios, it would be interesting to see the inter-site spin-spin correlation functions, which is however beyond the scope of the present study.

\section{Self-energy}

To investigate the nature of the transition, we plot in Fig. 4(a)-4(i) the raw data of the intra-orbital self-energy against the Matsubara frequency $\omega_{n}=(2 n+1) \pi T$ for $U / t=4.5,4.8,5.1$, and 5.4 at the temperature $T=0.05 t$. The self-energy is diagonal with respect to the orbital and two orbitals give the same self-energy, while it has a momentum dependence. Figures 4(a), 4(b), and 4(c) show the real part of the self-energy at the $(0,0)$ momentum $\operatorname{Re} \Sigma_{00}\left(i \omega_{n}\right)=-\operatorname{Re} \Sigma_{\pi \pi}\left(i \omega_{n}\right)$, its imaginary part $\operatorname{Im} \Sigma_{00}\left(i \omega_{n}\right)=\operatorname{Im} \Sigma_{\pi \pi}\left(i \omega_{n}\right)$, and the imaginary part of the $(\pi, 0)$ component $\operatorname{Im} \Sigma_{\pi 0}\left(i \omega_{n}\right)=\operatorname{Im} \Sigma_{0 \pi}\left(i \omega_{n}\right)$, respectively. Note that the real part of the $(\pi, 0)$ and $(0, \pi)$ components vanish due to the particle-hole symmetry.

First, we remark several features common to both $\mathrm{SU}(2)$ and $Z_{2}$ results. At the noninteracting limit $U / t=$ 0 , the Fermi surface exists at the $(\pi, 0)$ momentum while the $(0,0)-[(\pi, \pi)-]$ momentum state is occupied (unoccupied). In the Mott insulating state, this Fermi surface disappears at the $(\pi, 0)$ momentum due to the diver-

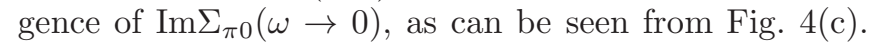
In the metallic region close to the Mott transition, the $(\pi, 0)$-momentum self-energy does not go to zero but to a finite value as $\omega \rightarrow 0$, which is a sign of a bad metal. To investigate whether this bad metallic behavior is intrinsic or it becomes a good metal at lower temperatures requires a huge computational cost and is in- 

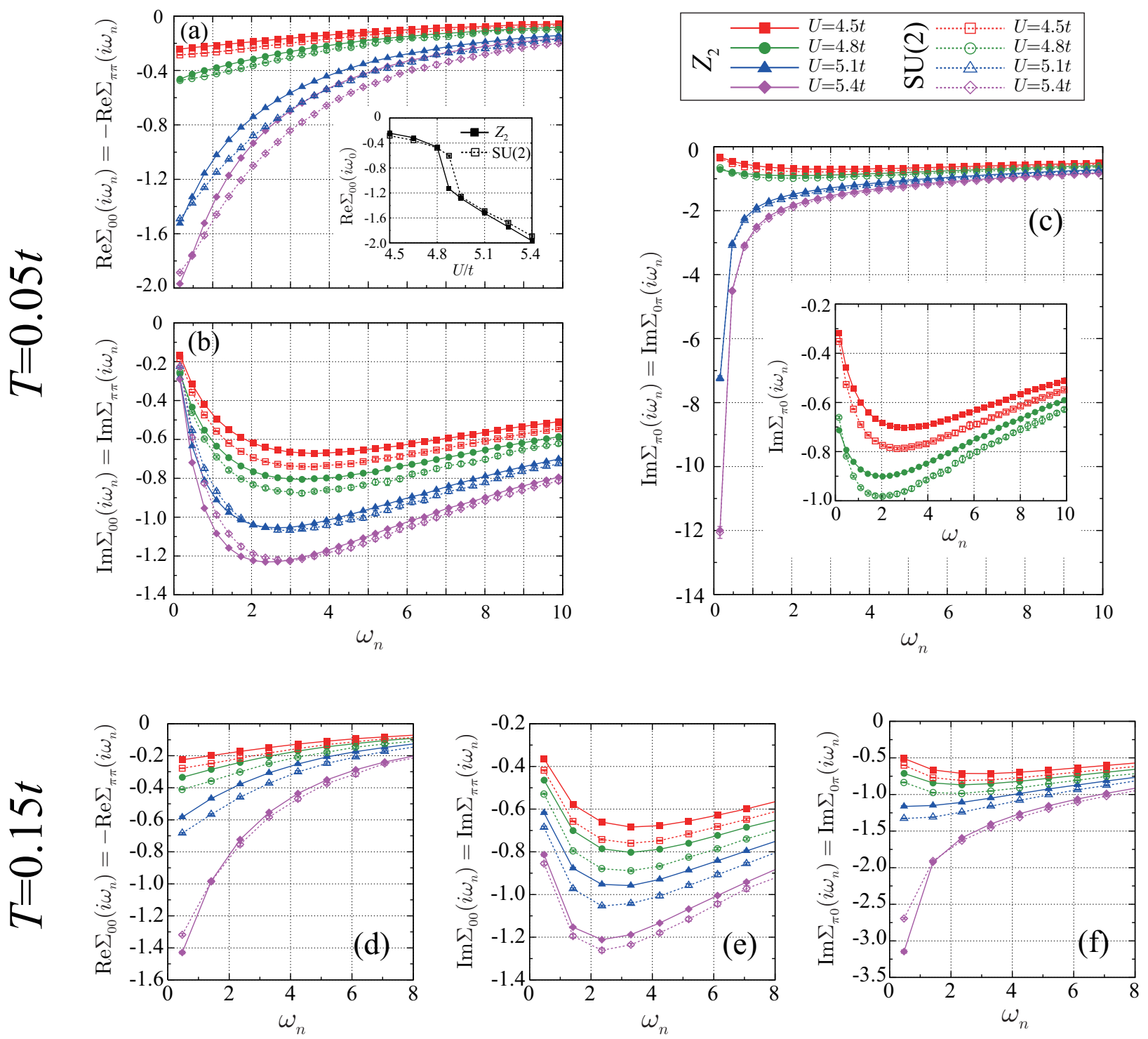

FIG. 4: (Color online) (a)-(c) The cDMFT results for the self-energies at $T=0.05 t$. The panels (a), (b), and (c) show the data for the real part of the self-energy at the $(0,0)$ momentum, $\operatorname{Re} \Sigma_{00}\left(i \omega_{n}\right)=-\operatorname{Re} \Sigma_{\pi \pi}\left(i \omega_{n}\right)$, its imaginary part, $\operatorname{Im} \Sigma_{00}\left(i \omega_{n}\right)=$ $\operatorname{Im} \Sigma_{\pi \pi}\left(i \omega_{n}\right)$, and the imaginary part of the self-energy at the $(\pi, 0)$ momentum, $\operatorname{Im} \Sigma_{\pi 0}\left(i \omega_{n}\right)=\operatorname{Im} \Sigma_{0 \pi}\left(i \omega_{n}\right)$, respectively. Due to the particle-hole symmetry, $\operatorname{Re} \Sigma_{\pi 0}\left(i \omega_{n}\right)=\operatorname{Re} \Sigma_{0 \pi}\left(i \omega_{n}\right)=0$. The inset of the panel (a) show the real part of the self-energy for the $(0,0)$ momentum at the first Matsubara frequency, $\operatorname{Re} \Sigma_{00}\left(i \omega_{0}\right)=-\operatorname{Re} \Sigma_{\pi \pi}\left(i \omega_{0}\right)$, as a function of the Hubbard interaction $U$. The data for $\operatorname{Im} \Sigma_{\pi 0}\left(i \omega_{n}\right)=\operatorname{Im} \Sigma_{0 \pi}\left(i \omega_{n}\right)$ at $U / t=4.5,4.8$ are zoomed in the inset of the panel (c). (d)-(f) The same as (a)-(c) but at $T=0.15 t$. The sizes of the error bars for the data for the $Z_{2}$-symmetric Hamiltonian are within those of the symbols. The lines are guides to the eye.

tractable at present. At the Mott transition, we see an abrupt change in $\operatorname{Re} \Sigma_{00}\left(i \omega_{n}\right)$ and $\operatorname{Re} \Sigma_{\pi \pi}\left(i \omega_{n}\right)$ (the inset of Fig. $4(a))$, which can also be used to determine the transition point. The similar change in $\operatorname{Re} \Sigma_{00}\left(i \omega_{n}\right)$ and $\operatorname{Re} \Sigma_{\pi \pi}\left(i \omega_{n}\right)$ is also seen in the cellular DMFT results for the 2D single-band Hubbard model on the square lattice $\stackrel{71}{\underline{11}}$ On the other hand, through the Mott transition, we do not find any anomaly in the imaginary part of the self-energy at $(0,0)$ and $(\pi, \pi)$ momentum $\left[\operatorname{Im} \Sigma_{00}\left(i \omega_{n}\right)\right.$ and $\left.\operatorname{Im} \Sigma_{\pi \pi}\left(i \omega_{n}\right)\right]$, where the Fermi surface does not exist even in the metallic state at small $U$.

We now turn to the comparison of the self-energy at $T=0.05 t$ between $\mathrm{SU}(2)$ and $Z_{2}$ cases at $U / t=$ $4.5,4.8,5.1$ and 5.4. For these values of interaction, the both types of Hamiltonian give a solution on the same side of the metal-insulator transition (see Fig. 3 and the inset of Fig. $4(\mathrm{a})$ ), and the difference in the resultant selfenergies is at most $\sim 20 \%$. A qualitative difference between $\mathrm{SU}(2)$ and $Z_{2}$ results can be seen only in the vicinity of the transition point: For example, for $U / t=4.875$ 
the $\mathrm{SU}(2)$-symmetric Hamiltonian still remains to give the metallic state while the $Z_{2}$-symmetric Hamiltonian incorrectly gives an insulating solution.

Finally, we show the self-energy at $T=0.15 t$ in Figs. 4(d), 4(e), and 4(f), where the crossover behavior from the metal to the insulator is seen. As is expected, the diverging behavior of $\operatorname{Im} \Sigma_{\pi 0}\left(i \omega_{n}\right)$ and $\operatorname{Im} \Sigma_{0 \pi}\left(i \omega_{n}\right)$ is much more moderate compared to that at $T=0.05 t$ (Figs. 4(a)-(c)). As for the difference between the results for the $\mathrm{SU}(2)$-symmetric Hamiltonian and those for the $Z_{2}$-symmetric Hamiltonian, generally the self-energies for the SU(2)-symmetric Hamiltonian are larger in magnitude, except for $\operatorname{Re} \Sigma_{00}\left(i \omega_{0}\right)$ and $\operatorname{Im} \Sigma_{\pi 0}\left(i \omega_{0}\right)$. However, the difference is at most $\sim 20 \%$. Similarly, we do not find any significant differences between the two types of Hamiltonian for the other parameter sets which have been studied in this paper. We however expect that these terms will give a substantial difference in two-particle quantities such as spin susceptibility (Ref. 65), which is left for future investigations.

\section{CONCLUSION}

We have incorporated the submatrix update into the CT-INT method and also developed the efficient sampling scheme, the double-vertex update, for the spin-flip and pair-hopping terms. Using the developed method, we have performed the cellular DMFT study for the 2D two-orbital Hubbard model on the square lattice. We have shown that the short-range spatial correlations significantly reduce the critical interaction strength for the Mott transition. The transition is induced by the divergence of the imaginary part of the $(\pi, 0)$-momentum self-energy and simultaneously we see the abrupt change in $\operatorname{Re} \Sigma_{00}\left(i \omega_{n}\right)=-\operatorname{Re} \Sigma_{\pi \pi}\left(i \omega_{n}\right)$. While we see the overestimate of the tendency toward the insulator in the $Z_{2^{-}}$ symmetric Hamiltonian, the difference in the critical interaction value between with and without the spin-flip and pair-hopping terms are smaller for the cDMFT results than that in the single-site DMFT case in the parameter region we have studied. When $J_{\mathrm{H}}$ is larger or a frustration is introduced, the difference might be more significant even in the cDMFT, which is an open problem.

The present scheme has established a firm starting point for the multi-orbital cDMFT study. Calculations at away from half-filling and/or for more than two orbitals are feasible. It is also interesting to study magnetism, superconductivity, orbital order, and so on, which we leave for future issues.

\section{Acknowledgments}

We would like to thank Philipp Werner, Giorgio Sangiovanni, and Nicolaus Parragh for fruitful discussions. This work was supported by Funding Program for World-
Leading Innovative R\&D on Science and Technology (FIRST program) on "Quantum Science on Strong Correlation". Y. N. is supported by the Grant-in-Aid from JSPS (Grant No. 12J08652). The calculations were performed at the Supercomputer Center, ISSP, University of Tokyo.

\section{Appendix A: Calculation of the Green's function matrix $G$}

The Green's function matrix $G$ (or $\tilde{G}$ ) in Eq. (22) is related to the $A$ matrix by $G_{\sigma}=A_{\sigma}^{-1} G_{0 \sigma}$. When the configuration $C_{n}^{\prime}=\left\{\left(s_{1}^{\prime}, \tau_{1}\right), \cdots,\left(s_{n}^{\prime}, \tau_{n}\right)\right\}$ differs from $C_{n}=\left\{\left(s_{1}, \tau_{1}\right), \cdots,\left(s_{n}, \tau_{n}\right)\right\}$ in only the spin orientation, $A_{\sigma}^{\prime}\left(C_{n}^{\prime}\right)$ is related to $A_{\sigma}\left(C_{n}\right)$ via the Dyson equation

$$
A_{\sigma}^{\prime-1}=A_{\sigma}^{-1}+\left(G_{\sigma}-I\right) \Lambda_{\sigma} A_{\sigma}^{\prime-1} .
$$

Here $I$ is an $n \times n$ identity matrix and

$$
\left[\Lambda_{\sigma}\right]_{i j}=\delta_{i j} \frac{f_{\sigma}\left(s_{i}^{\prime}\right)-f_{\sigma}\left(s_{i}\right)}{f_{\sigma}\left(s_{i}\right)} .
$$

By setting $s_{i}^{\prime}=0$ for all $i$ in Eq. A1, we obtain

$$
\left(f_{\sigma}\left(s_{j}\right)-1\right)\left[G_{\sigma}\right]_{i j}=f_{\sigma}\left(s_{j}\right)\left[A_{\sigma}^{-1}\right]_{i j}+\delta_{i j} .
$$

If $s_{j} \neq 0$, we can use this efficient formula to calculate $\left[G_{\sigma}\right]_{i j}$, otherwise, we need to compute $\left[G_{\sigma}\right]_{i j}$ directly by

$$
\left[G_{\sigma}\right]_{i j}=\left[A_{\sigma}^{-1}\right]_{i k}\left[G_{0 \sigma}\right]_{k j} .
$$

\section{Appendix B: Absence of the sign problem within the double-vertex update}

Here, we prove that the negative signs are absent within the double-vertex update in the two-orbital systems, in a way similar to that employed in Ref. 61 for the single-orbital Hubbard model. We first consider the case of $\delta_{3}=0$ in Eq. (41). Following Refs. 61 and 72, we introduce a chain representation for the non-interacting part of the impurity Hamiltonian,

$\tilde{\mathcal{H}}_{0}=\sum_{i, \sigma} \sum_{r=0}^{\infty}\left[\tilde{\epsilon}_{i r} \hat{d}_{i, r}^{\sigma \dagger} \hat{d}_{i, r}^{\sigma}-t_{i r}\left(\hat{d}_{i, r+1}^{\sigma \dagger} \hat{d}_{i, r}^{\sigma}+\hat{d}_{i, r}^{\sigma \dagger} \hat{d}_{i, r+1}^{\sigma}\right)\right]$

where $\hat{d}_{i, r}^{\sigma \dagger}\left(\hat{d}_{i, r}^{\sigma}\right)$ is the creation (annihilation) operator for the orbital $i$ and the site $r . r=0$ denotes the impurity site, and hence $\hat{d}_{i, 0}^{\sigma}=\hat{c}_{i \sigma}$ and $\tilde{\epsilon}_{i 0}=-\tilde{\mu} . r \geq 1$ denotes an infinite chain of the bath sites attached to the impurity site. With a proper choice of the gauge, all the hopping parameters $t_{i r}$ can be taken to be non-negative, i.e., $t_{i r} \geq$ 0 . The weight for a configuration $C_{n}$ is

$$
\begin{aligned}
W\left(C_{n}\right)=\operatorname{Tr} & {\left[e^{-\left(\beta-\tau_{n}\right) \tilde{\mathcal{H}}_{0}} V\left(\kappa_{n}, s_{n}\right) e^{-\left(\tau_{n}-\tau_{n-1}\right) \tilde{\mathcal{H}}_{0}}\right.} \\
& \left.\times V\left(\kappa_{n-1}, s_{n-1}\right) \cdots e^{-\tau_{1} \tilde{\mathcal{H}}_{0}}\right]
\end{aligned}
$$


where $V\left(\kappa_{p}, s_{p}\right)$ represents a vertex of the type $\kappa_{p}$ and of the auxiliary spin $s_{p}$, which is inserted at the imaginary time $\tau_{p}$ : For example, for one of the spin-flip terms $\left(\kappa_{p}=\right.$ 7) with $\delta_{3}=0$, it is written as

$$
V\left(\kappa_{p}, s_{p}\right)=-\frac{J_{\mathrm{H}} d \tau}{4} \hat{c}_{1 \uparrow}^{\dagger}\left(\tau_{p}\right) \hat{c}_{2 \uparrow}\left(\tau_{p}\right) \hat{c}_{2 \downarrow}^{\dagger}\left(\tau_{p}\right) \hat{c}_{1 \downarrow}\left(\tau_{p}\right) .
$$

On the chain basis, it has been shown that all the elements of the $e^{-\tau \tilde{\mathcal{H}}_{0}}$ matrix are non-negative, which is also true for the density-type vertices $V(\kappa, s)$ irrespective to the spin orientation $s= \pm 1 .^{61,72}$ On the other hand, for the non-density-type vertex, it is easy to see that all the elements of the $-V(\kappa, s)$ matrix are non-negative. Since the non-density-type vertices always appear in pair within the double-vertex update, the product involving the pair of the vertices $V(\kappa, s)$ is always non-negative. Then, the weight $W\left(C_{n}\right)$ turns out to be the trace of the product of the matrices with non-negative elements, and therefore it is non-negative. Although we need a finite $\delta_{3}$ for the submatrix update, a similar pair cancellation of the negative factors of the vertices will work as far as $\delta_{3}$ is small.
* Electronic address: nomura@moegi.t.u-tokyo.ac.jp

1 M. Imada, A. Fujimori, and Y. Tokura, Rev. Mod. Phys. 70, 1039 (1998), URL http://link.aps.org/doi/10.1103/RevModPhys.70.1039

2 H. Tasaki, Progress of Theoretical Physics 99, 489 (1998), http://ptp.oxfordjournals.org/content/99/4/489.full.pdf+html, Millis, Phys. Rev. B 80, 045120 (2009), URL URL http://ptp.oxfordjournals.org/content/99/4/489.abst/tetdt:://link .aps.org/doi/10.1103/PhysRevB.80.045120.

3 A. Georges, G. Kotliar, W. Krauth, and M. J. Rozenberg, Rev. Mod. Phys. 68, 13 (1996), URL http://link.aps.org/doi/10.1103/RevModPhys.68.13.

4 T. Maier, M. Jarrell, T. Pruschke, and M. H. Hettler, Rev. Mod. Phys. 77, 1027 (2005), URL http://link.aps.org/doi/10.1103/RevModPhys.77.1027

5 G. Kotliar, S. Y. Savrasov, G. Pálsson, and G. Biroli, Phys. Rev. Lett. 87, 186401 (2001), URL http://link.aps.org/doi/10.1103/PhysRevLett.87.186401

6 A. I. Lichtenstein and M. I. Katsnelson, Phys. Rev. B 62, R9283 (2000), URL http://link.aps.org/doi/10.1103/PhysRevB.62.R9283.

7 M. Potthoff, M. Aichhorn, and C. Dahnken, Phys. Rev. Lett. 91, 206402 (2003), URL http://link.aps.org/doi/10.1103/PhysRevLett.91.206402

8 C. Huscroft, M. Jarrell, T. Maier, S. Moukouri, and A. N. Tahvildarzadeh, Phys. Rev. Lett. 86, 139 (2001), URL http://link.aps.org/doi/10.1103/PhysRevLett.86.139

9 T. A. Maier, T. Pruschke, and M. Jarrell, Phys. Rev. B 66, 075102 (2002), URL http://link.aps.org/doi/10.1103/PhysRevB.66.075102

10 B. Kyung, S. S. Kancharla, D. Sénéchal, A.M. S. Tremblay, M. Civelli, and G. Kotliar, Phys. Rev. B 73, 165114 (2006), URL http://link.aps.org/doi/10.1103/PhysRevB.73.165114

11 T. D. Stanescu and G. Kotliar, Phys. Rev. B $\mathbf{7 4}, \quad 125110 \quad$ (2006), URL http://link.aps.org/doi/10.1103/PhysRevB.74.125110

12 Y. Z. Zhang and M. Imada, Phys. Rev. B 76, $045108 \quad$ (2007), URL http://link.aps.org/doi/10.1103/PhysRevB.76.045108

13 M. Civelli, M. Capone, S. S. Kancharla, O. Parcollet, and G. Kotliar, Phys. Rev. Lett. 95, 106402 (2005), URL http://link.aps.org/doi/10.1103/PhysRevLett.95.106402

14 A. Macridin, M. Jarrell, T. Maier, P. R. C. Kent, and E. D'Azevedo, Phys. Rev. Lett. 97, 036401 (2006), URL http://link.aps.org/doi/10.1103/PhysRevLett.97.036401

15 M. Civelli, M. Capone, A. Georges, K. Haule, O. Parcollet, T. D. Stanescu, and G. Kotliar, Phys. Rev. Lett. 100, 046402 (2008), URL
http://link.aps.org/doi/10.1103/PhysRevLett.100.046402.

16 S. Sakai, Y. Motome, and M. Imada, Phys. Rev. Lett. 102, 056404 (2009), URL http://link.aps.org/doi/10.1103/PhysRevLett.102.056404.

17 P. Werner, E. Gull, O. Parcollet, and A. J. $\begin{array}{lcccc}\text { A. Liebsch and N.-H. Tong, Phys. } & \end{array}$ http://link.aps.org/doi/10.1103/PhysRevB.80.165126

19 N. Lin, E. Gull, and A. J. Millis, Phys. Rev. B 82, 045104 (2010), URL http://link.aps.org/doi/10.1103/PhysRevB.82.045104,

20 S. Sakai, Y. Motome, and M. Imada, Phys. Rev. B 82, 134505 (2010), URL http://link.aps.org/doi/10.1103/PhysRevB.82.134505.

21 S. Sakai, G. Sangiovanni, M. Civelli, Y. Motome, K. Held, and M. Imada, Phys. Rev. B 85, 035102 (2012), URL http://link.aps.org/doi/10.1103/PhysRevB.85.035102,

22 N. Lin, E. Gull, and A. J. Millis, Phys. Rev. Lett. 109, 106401 (2012), URL http://link.aps.org/doi/10.1103/PhysRevLett.109.106401. 23 G. Sordi, P. Sémon, K. Haule, and A.-M. S. Tremblay, Sci. Rep. 2, $547 \quad$ (2003), URL http://dx.doi.org/10.1038/srep00547

24 S. Sakai, S. Blanc, M. Civelli, Y. Gallais, M. Cazayous, M.-A. Méasson, J. S. Wen, Z. J. Xu, G. D. Gu, G. Sangiovanni, et al., Phys. Rev. Lett. 111, 107001 (2013), URL http://link.aps.org/doi/10.1103/PhysRevLett.111.107001.

25 S. Okamoto, D. Sénéchal, M. Civelli, and A.-M. S. Tremblay, Phys. Rev. B 82, 180511 (2010), URL http://link.aps.org/doi/10.1103/PhysRevB.82.180511.

26 S. Okamoto and N. Furukawa, Phys. Rev. B 86, $094522 \quad$ (2012), URL http://link.aps.org/doi/10.1103/PhysRevB.86.094522.

27 D. Sénéchal, P.-L. Lavertu, M.-A. Marois, and A.M. S. Tremblay, Phys. Rev. Lett. 94, 156404 (2005), URL http://link.aps.org/doi/10.1103/PhysRevLett.94.156404 and J. B. White, Phys. Rev. Lett. 95, 237001 (2005), URL http://link.aps.org/doi/10.1103/PhysRevLett.95.237001

M. Aichhorn, E. Arrigoni, M. Potthoff, and W. Hanke, Phys. Rev. B 74, 024508 (2006), URL http://link.aps.org/doi/10.1103/PhysRevB.74.024508. M. Aichhorn, E. Arrigoni, Z. B. Huang, and W. Hanke, Phys. Rev. Lett. 99, 257002 (2007), URL http://link.aps.org/doi/10.1103/PhysRevLett.99.257002
28 T. A. Maier, M. Jarrell, T. C. Schulthess, P. R. C. Kent, 
31 M. Capone and G. Kotliar, Phys. Rev. B $\mathbf{7 4}, 054513 \quad$ (2006), URL http://link.aps.org/doi/10.1103/PhysRevB.74.054513

32 K. Haule and G. Kotliar, Phys. Rev. B $\quad \mathbf{7 6}, \quad 104509 \quad$ (2007), URL http://link.aps.org/doi/10.1103/PhysRevB.76.104509 33 S. Okamoto and T. A. Maier, Phys. Rev. Lett. 101, 156401 (2008), URL http://link.aps.org/doi/10.1103/PhysRevLett.101.156401

34 S. S. Kancharla, B. Kyung, D. Sénéchal, M. Civelli, M. Capone, G. Kotliar, and A.-M. S. Tremblay, Phys. Rev. B 77, 184516 (2008), URL http://link.aps.org/doi/10.1103/PhysRevB.77.184516

35 M. Civelli, Phys. Rev. B 79, 195113 (2009), URL http://link.aps.org/doi/10.1103/PhysRevB.79.195113

36 E. Khatami, A. Macridin, and M. Jarrell, Phys. Rev. B 80, 172505 (2009), URL http://link.aps.org/doi/10.1103/PhysRevB.80.172505

37 B. Kyung, D. Sénéchal, and A.-M. S. Tremblay, Phys. Rev. B 80, 205109 (2009), URL http://link.aps.org/doi/10.1103/PhysRevB.80.205109

38 M. Civelli, Phys. Rev. Lett. 103, 136402 (2009), URL http://link.aps.org/doi/10.1103/PhysRevLett.103.136402

39 G. Sordi, P. Sémon, K. Haule, and A.-M. S. Tremblay, Phys. Rev. Lett. 108, 216401 (2012), URL http://link.aps.org/doi/10.1103/PhysRevLett.108.216401

40 E. Gull, O. Parcollet, and A. J. Millis, Phys. Rev. Lett. 110, 216405 (2013), URL http://link.aps.org/doi/10.1103/PhysRevLett.110.216405

41 K.-S. Chen, Z. Y. Meng, S.-X. Yang, T. Pruschke, J. Moreno, and M. Jarrell, arXiv.1308.5946 (2013), URL http://arxiv.org/abs/1308.5946.

$42 \mathrm{~S}$. Okamoto and T. A. Maier, Phys. Rev. B 81, $214525 \quad$ (2010), URL http://link.aps.org/doi/10.1103/PhysRevB.81.214525

43 H. Sakakibara, H. Usui, K. Kuroki, R. Arita, and H. Aoki, Phys. Rev. Lett. 105, 057003 (2010), URL http://link.aps.org/doi/10.1103/PhysRevLett.105.057003

44 H. Sakakibara, H. Usui, K. Kuroki, R. Arita, and H. Aoki, Phys. Rev. B 85, 064501 (2012), URL http://link.aps.org/doi/10.1103/PhysRevB.85.064501

45 H. Miyahara, R. Arita, and H. Ikeda, Phys. Rev. B 87, 045113 (2013), URL http://link.aps.org/doi/10.1103/PhysRevB.87.045113

46 T. Kita, T. Ohashi, and S.-i. Suga, Phys. Rev. B 79, 245128 (2009), URL http://link.aps.org/doi/10.1103/PhysRevB.79.245128

47 J. E. Hirsch and R. M. Fye, Phys. Rev. Lett. 56, $2521 \quad$ (1986), URL http://link.aps.org/doi/10.1103/PhysRevLett.56.2521

48 A. I. Poteryaev, A. I. Lichtenstein, and G. Kotliar, Phys. Rev. Lett. 93, 086401 (2004), URL http://link.aps.org/doi/10.1103/PhysRevLett.93.086401

49 E. Gull, A. J. Millis, A. I. Lichtenstein, A. N. Rubtsov, M. Troyer, and P. Werner, Rev. Mod. Phys. 83, 349 (2011), URL http://link.aps.org/doi/10.1103/RevModPhys.83.349.

50 S. M. A. Rombouts, K. Heyde, and N. Jachowicz, Phys. Rev. Lett. 82, 4155 (1999), URL http://link.aps.org/doi/10.1103/PhysRevLett.82.4155

51 H. Lee, Y.-Z. Zhang, H. O. Jeschke, R. Valentí, and H. Monien, Phys. Rev. Lett. 104, 026402 (2010), URL http://link.aps.org/doi/10.1103/PhysRevLett.104.026402

52 A. N. Rubtsov, V. V. Savkin, and A. I. Licht- enstein, Phys. Rev. B 72, 035122 (2005), URL http://link.aps.org/doi/10.1103/PhysRevB.72.035122.

53 A. Rubtsov and A. Lichtenstein, Journal of Experimental and Theoretical Physics Letters 80, 61 (2004), ISSN 00213640, URL http://dx.doi.org/10.1134/1.1800216

${ }^{54}$ F. F. Assaad and T. C. Lang, Phys. Rev. B $\quad \mathbf{7 6}, \quad 035116 \quad$ (2007), URL http://link.aps.org/doi/10.1103/PhysRevB.76.035116. A. E. Antipov, I. S. Krivenko, V. I. Anisimov, A. I. Lichtenstein, and A. N. Rubtsov, Phys. Rev. B 86, 155107 (2012), URL http://link.aps.org/doi/10.1103/PhysRevB.86.155107.

56 P. Werner, A. Comanac, L. de' Medici, M. Troyer, and A. J. Millis, Phys. Rev. Lett. 97, 076405 (2006), URL http://link.aps.org/doi/10.1103/PhysRevLett.97.076405

57 N. Parragh, A. Toschi, K. Held, and G. Sangiovanni, Phys. Rev. B 86, 155158 (2012), URL http://link.aps.org/doi/10.1103/PhysRevB.86.155158.

58 G. Li and W. Hanke, Phys. Rev. B 85, 115103 (2012), URL http://link.aps.org/doi/10.1103/PhysRevB.85.115103.

59 P. K. V. V. Nukala, T. A. Maier, M. S. Summers, G. Alvarez, and T. C. Schulthess, Phys. Rev. B 80, 195111 (2009), URL http://link.aps.org/doi/10.1103/PhysRevB.80.195111. 60 E. Gull, P. Staar, S. Fuchs, P. Nukala, M. S. Summers, T. Pruschke, T. C. Schulthess, and T. Maier, Phys. Rev. B 83, 075122 (2011), URL http://link.aps.org/doi/10.1103/PhysRevB.83.075122. P. Werner, Lecture notes on DMFT, ETH Zurich (2011).

E. Gorelov, T. O. Wehling, A. N. Rubtsov, M. I. Katsnelson, and A. I. Lichtenstein, Phys. Rev. B 80, 155132 (2009), URL http://link.aps.org/doi/10.1103/PhysRevB.80.155132,

63 K. Held and D. Vollhardt, Eur. Phys. J. B 5, 473 (1998), URL http://dx.doi.org/10.1007/s100510050468

64 S. Sakai, R. Arita, and H. Aoki, Phys. Rev. B 70, 172504 (2004), URL http://link.aps.org/doi/10.1103/PhysRevB.70.172504, S. Sakai, R. Arita, K. Held, and H. Aoki, Phys. Rev. B 74, $155102 \quad$ (2006), URL http://link.aps.org/doi/10.1103/PhysRevB.74.155102

66 J. E. Han, Phys. Rev. B 70, 054513 (2004), URL http://link.aps.org/doi/10.1103/PhysRevB.70.054513,

67 L. Boehnke, H. Hafermann, M. Ferrero, F. Lechermann, and O. Parcollet, Phys. Rev. B 84, 075145 (2011), URL http://link.aps.org/doi/10.1103/PhysRevB.84.075145

68 Y. Nomura, M. Kaltak, K. Nakamura, C. Taranto, S. Sakai, A. Toschi, R. Arita, K. Held, G. Kresse, and M. Imada, Phys. Rev. B 86, 085117 (2012), URL http://link.aps.org/doi/10.1103/PhysRevB.86.085117

69 L. Vaugier, H. Jiang, and S. Biermann, Phys. Rev. B 86, 165105 (2012), URL http://link.aps.org/doi/10.1103/PhysRevB.86.165105, T. Pruschke and R. Bulla, The European Physical Journal B - Condensed Matter and Complex Systems 44, 217 (2005), ISSN 1434-6028, URL http://dx.doi.org/10.1140/epjb/e2005-00117-4

71 H. Park, K. Haule, and G. Kotliar, Phys. Rev. Lett. 101, 186403 (2008), URL http://link.aps.org/doi/10.1103/PhysRevLett.101.186403.

72 J. Yoo, S. Chandrasekharan, R. K. Kaul, D. Ullmo, and H. U. Baranger, Journal of Physics A: Mathematical and General 38, 10307 (2005), URL http://stacks.iop.org/0305-4470/38/i=48/a=004 